\title{
Suppressive Effect of Tritoqualine on Enzyme Leakage Induced by D-Galactosamine in Rat Primary-Cultured Hepatocytes
}

\author{
Satoshi YUASA, Yoshihide NAKAO and Kohei UMEZU \\ Pharmaceuticals Laboratory, Research Center, Mitsubishi Chemical Ind., Ltd., \\ Yokohama 227, Japan \\ Accepted November 15, 1986
}

\begin{abstract}
Tritoqualine (TRQ) was previously reported to suppress enzyme leakage and lipid peroxidation induced by carbon tetrachloride in isolated hepatocytes. In the present study, we investigated the effect of TRQ on enzyme leakage from rat primary-cultured hepatocytes using D-galactosamine (GalN) which causes hepatic injury without lipid peroxidation. Leakage of GPT and GPT was significantly increased at $18 \mathrm{hr}$ after GalN addition, being saturated at $42-50 \mathrm{hr}$. This enzyme leakage was suppressed dose-dependently by TRO at $42 \mathrm{hr}$, but not by vitamin $E$. These results suggest that TRQ shows a suppressive effect on enzyme leakage from hepatocytes independently of its inhibitory action on lipid peroxidation.
\end{abstract}

Since tritoqualine (TRQ: $( \pm)-\left(R^{*}\right)-7$ amino-4,5,6-triethoxy-3-[(R*)-5,6,7,8-tetrahydro-4-methoxy-6-methyl-1,3-dioxolo [4,5g] isoquinolin-5-yl] phthalide) markedly suppressed an increase of serum transaminases induced by a single administration of carbon tetrachloride $\left(\mathrm{CCl}_{4}\right)$, GalN or allylalcohol to rats, protection of the hepatocyte membrane was presumed to be one of the pharmacological effects of TRO (1). In addition, TRO showed suppressive effects on lipid peroxidation and enzyme leakage caused by $\mathrm{CCl}_{4}$ addition in freshly isolated rat hepatocytes. Therefore, it was thought that the membrane protecting action of TRQ was derived from its inhibition of lipid peroxidation (2). Vitamin $\mathrm{E}$, an endogeneous antioxidant, showed almost a similar inhibitory action on lipid peroxidation, but the suppressive effect of TRQ on enzyme release was more than twice as strong as that of vitamin $E$ (2). These results have suggested that TRO had not only an antioxidative action but also another action involved in membrane protection (2). The present study was designed to investigate the effect of TRO on enzyme leakage from rat hepatocytes using GalN which is known to show hepatotoxicity without lipid peroxidation (3).

\section{Materials and Methods}

Isolated hepatocytes were prepared from male Wistar rats (Japan Laboratory Animals Inc.) weighing $200-250 \mathrm{~g}$ by collagenase perfusion, as described previously (2). The hepatocytes were suspended in William's E medium (Flow) supplemented with 10\% new born calf serum (Mitsubishi Chemical Ind., Ltd.), $100 \mathrm{U} / \mathrm{ml}$ penicillin (Flow), 100 $\mu \mathrm{g} / \mathrm{ml}$ streptomycin (Flow). $0.25 \mu \mathrm{g} / \mathrm{ml}$ fungizone (Flow). $10^{-6} \mathrm{M}$ dexamethasone (Sigma) and $10^{-8} \mathrm{M}$ insulin (Sigma) and inoculated at a density of $5 \times 10^{5}$ cells $/ 1 \mathrm{ml} /$ plate (9.6 $\mathrm{cm}^{2}$. Falcon). After incubation in a humidified atmosphere of $5 \% \mathrm{CO}_{2}-95 \%$ air at $37^{\circ} \mathrm{C}$ for $90 \mathrm{~min}$ to allow attachment of the cells $(4,5)$, the culture medium was discarded to remove unattached cells. The hepatocytes were then incubated in a fresh medium containing $1 \mathrm{mM}$ GalN (Sigma). At 2, 18, 26, 42 and $50 \mathrm{hr}$ after GalN addition, the supernatant of the culture medium was separated by centrifugation. GPT (EC 2.6.1.2) and GOT (EC 2.6.1.1) activities in these supernatants were measured using commercial test kits (GPT-UV and GOT-UV. Wako Pure Chemicals). As a marker of lipid peroxidation, the amount of malondialdehyde (MDA) 
present in the plate was determined using the TCA (trichloroacetic acid) soluble fractions (6).

In order to investigate the effects of TRQ. $10 \mu l$ of TRO (Mitsubishi Chemical Ind., Ltd.) dissolved in $0.1 \mathrm{~N} \mathrm{HCl}$ was added to the culture medium simultaneously with GalN. Vitamin E ( $\alpha$-tocopherol, Tokyo Kasei Kogyo) and glycyrrhetinic acid (18 $\beta$. Sigma) were dissolved in DMSO (Junsei Chemical) and added in the same way. Glycyrrhizin (SNMC: $0.2 \%$ glycyrrhizin, $0.1 \%$ cysteine, $2 \%$ glycine. Minophagen Seiyaku Honpo) was diluted by PBS (9.6 mM phosphate buffer, $\mathrm{pH}$ 7.4. $137 \mathrm{mM} \mathrm{NaCl}, 2.7 \mathrm{mM} \mathrm{KCl}$ ) and added at $50 \mu \mathrm{l} /$ plate. At $42 \mathrm{hr}$ after GalN addition, the supernatant of the culture medium was separated by centrifugation at $10,000 \times \mathrm{g}$, and the activity of GPT and GOT were measured similarly.

\section{Results}

When freshly isolated hepatocytes were incubated with GalN for $5 \mathrm{hr}$, enzyme leakage was not found (data not shown). The present investigation was carried out in a primary-cultured system. As shown in Fig. 1. enzyme activities leaked from hepatocytes were increased after $18 \mathrm{hr}$ of $1 \mathrm{mM} \mathrm{GalN}$ addition and almost saturated at $42 \mathrm{hr}$ (Fig. 1). The leakage patterns of GPT and GOT were similar to each other, but the activity of leaked GOT was about 7 times as high as that of GPT. The enzyme leakage was suppressed dose-dependently at $42 \mathrm{hr}$ of incubation with TRO (Fig. 2). Table 1 shows the suppressive effect of TRO, compared with the other compounds, Vitamin E (100 $u \mathrm{M})$ and glycyrrhizin $(122 \mu \mathrm{M})$ were ineffective, but $1 \mathrm{mM}$ of glycyrrhetinic acid suppressed only GPT leakage.

\section{Discussion}

In the previous paper (2), it was suggested that the membrane protecting action of TRO against $\mathrm{CCl}_{4}$-induced hepatic injury was derived from its antioxidative activity, but that TRQ had also another action which contributed to membrane protection. In order to confirm this other action of TRQ, it is necessary to use a different hepatotoxin which does not induce lipid peroxidation. Watanabe et al. (3) reported that GalN caused enzyme leakage from the liver in vivo without lipid peroxidation. We actually confirmed that GalN did not increase the amount of lipid peroxides in rat liver in vivo (Umezu et al., unpublished data). Therefore, the effect of TRO was investigated in isolated hepatocytes using GalN.

It has been thought that this enzyme leakage represented the greater part of the

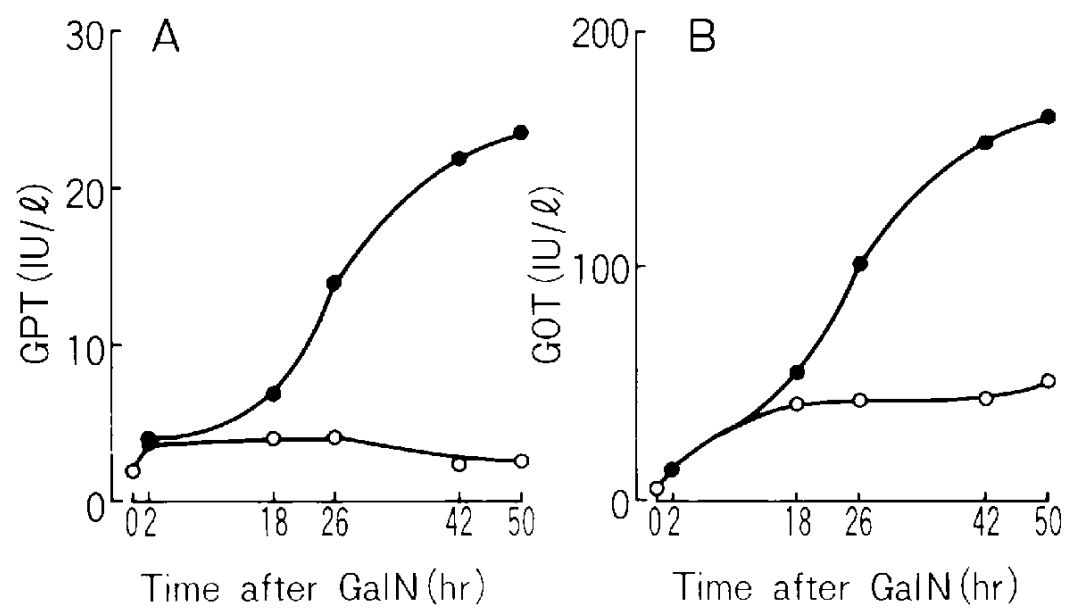

Fig. 1. Time variation of GPT and GOT leakage from primary cultured hepatocytes after treatment with $1 \mathrm{mM}$ GalN. Ninety min after plating. hepatocytes were exposed to $1 \mathrm{mM} \mathrm{GalN}$ in a fresh medium. Enzyme activities in the medium were measured at the times indicated. The data provide triplicate incubations (S.E.M. $<10 \%$ ). GalN addition, O no addition. A: leakage of GPT, B: leakage of GOT. 

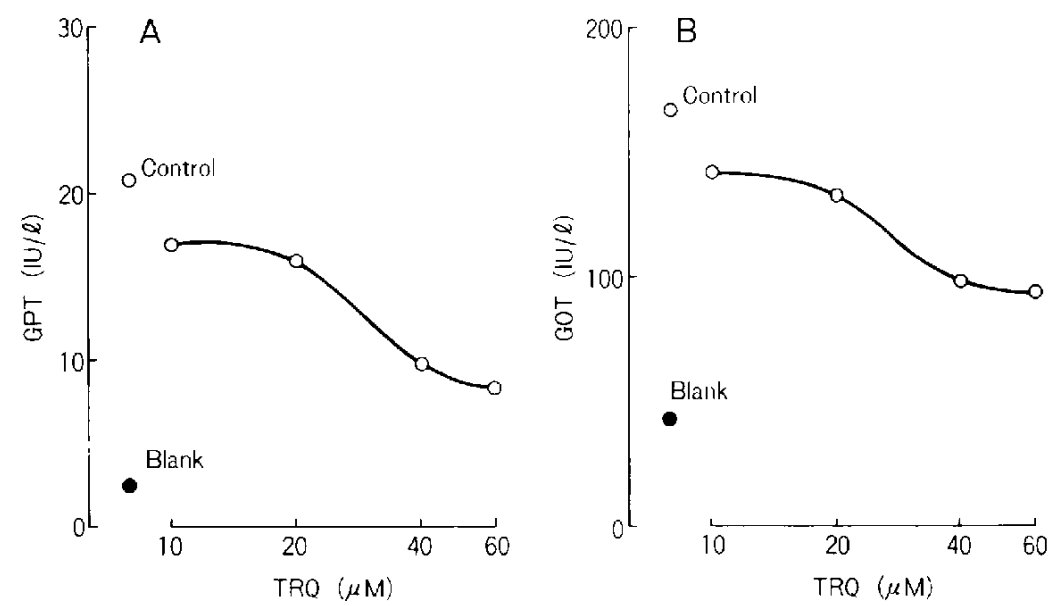

Fig. 2. Effect of TRO on galactosamine-induced enzyme leakage from primary-cultured hepatocytes. Ninety min after plating, hepatocytes were exposed to $1 \mathrm{mM} \mathrm{GalN}$ in a fresh medium and TRQ (dissolved in $0.1 \mathrm{~N} \mathrm{HCl}$ ) was simultaneously added. After $42 \mathrm{hr}$ of incubation, enzyme activities in the medium were measured. The data are from triplicate incubations (S.E.M. <10\%), Blank: no addition. Control: $0.1 \mathrm{~N} \mathrm{HCl}$ addition. A: leakage of GPT, B: leakage of GOT.

Table 1. Effect of various compounds on galactosamine -induced enzyme leakage from primary-cultured hepatocytes

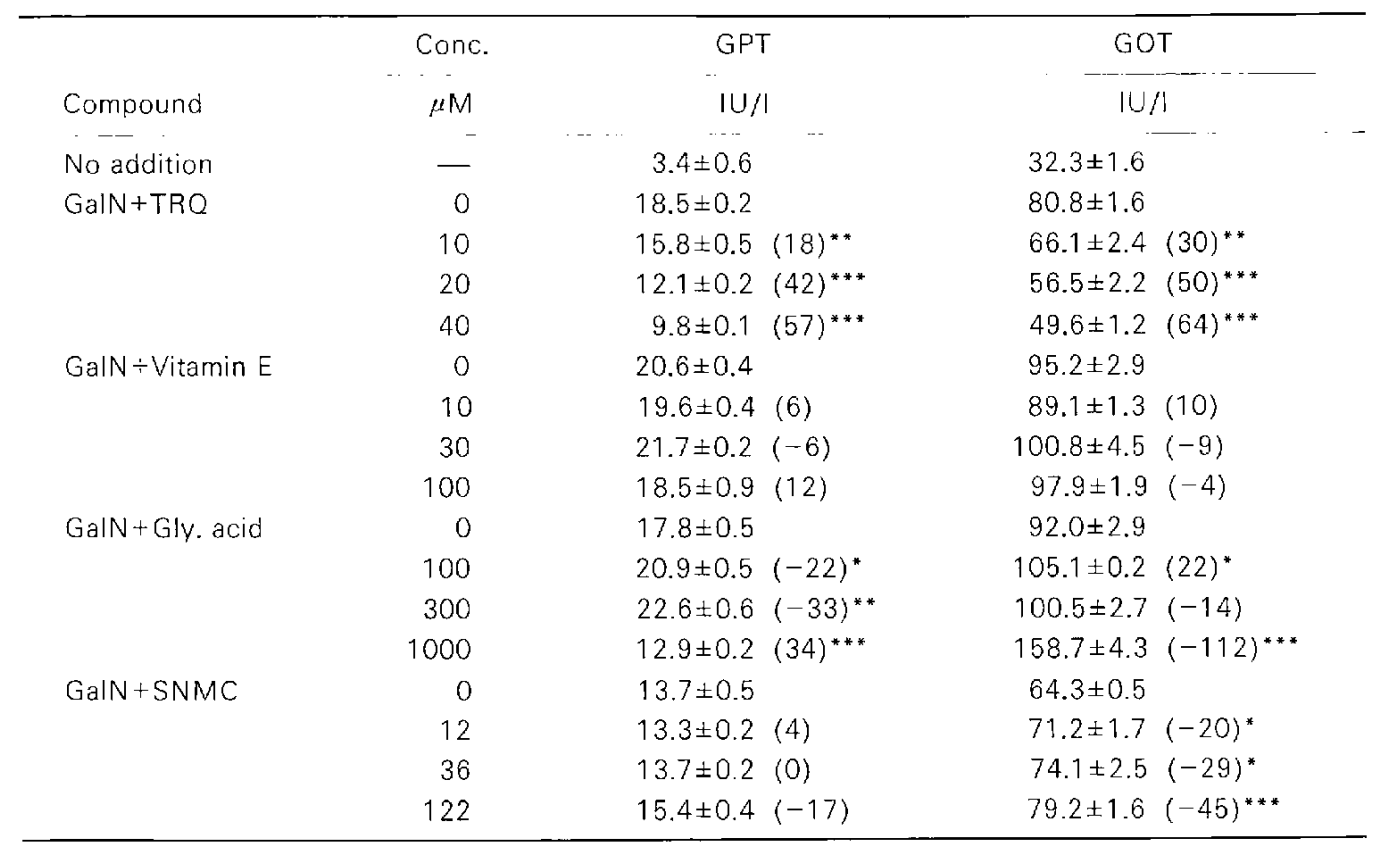

Ninety min after plating, hepatocytes were exposed to $1 \mathrm{mM} \mathrm{GalN}$ in fresh medium and test compounds were simultaneously added. After $42 \mathrm{hr}$ of incubation, enzyme activities in the medium were measured. Each value is the mean \pm S.E.M. of three plates. Values in parentheses indicate percentage figures for the reduction of enzyme activity. ( $\left.{ }^{*}\right) P<0.05,\left({ }^{* *}\right) P<0.01$ and $\left({ }^{* *}\right) P<0.001$, compared with each vehicle-treated control (Student's t-test). Gly, acid: glycyrrhetinic acid. SNMC: glycyrhizin mixture. 
necrosis in hepatocytes $(4,5)$, and its characteristics closely resembled liver injury in vivo (7). The effect of TRO on enzyme leakage was investigated at $42 \mathrm{hr}$ after GalN addition. when hepatocyte injury became very severe. TRQ suppressed, dosedependently, both the GPT and GOT leakages, as shown in Fig. 2.

Table 1 shows a comparison between TRQ and the other compounds. Vitamin E did not suppress enzyme leakage at all, even though the inhibition of lipid peroxidation caused by $\mathrm{CCl}_{4}$ was similar to that by TRQ. This result indicates that the liver injury in vivo caused by $\mathrm{GalN}$ is independent of lipid peroxidation. In fact. MDA was not produced in this culture system ( $<1$ nmole/plate) with or without GalN (data not shown). That is, TRQ was confirmed to suppress the enzyme leakage without inhibitory action on lipid peroxidation. Yoshikawa et al. (8) found that liver disorder induced by GalN was remarkable in vitamin $E$ deficient rats and slight in those given vitamin $E$ supplements. Consequently, they reported that vitamin $E$ prevented liver disorders by inhibiting lipid peroxidation. However, they thought that lipid peroxidation was not the primary cause of liver impairment but gave rise to subsequent damage to the liver. Therefore, the above results were thought to support their hypothesis.

Kiso et al. (4) reported that glycyrrhetinic acid at 0.1 and $1.0 \mu \mathrm{g} / \mathrm{ml}$ suppressed the leakage of GPT. In the present study, it suppressed GPT leakage at a concentration of $1 \mathrm{mM}$ (about $0.5 \mu \mathrm{g} / \mathrm{ml}$ ); however, its acceleration of GOT leakage was observed at the same concentration. In addition, glycyrrhizin (SNMC), which was used clinically as an antihepatotoxic agent, was ineffective in this culture system. Therefore, it was suggested that TRO has a different mechanism from glycyrrhizin for protection of the hepatocyte membrane.

According to Farber (9), $\mathrm{CCl}_{4}$ and $\mathrm{GalN}$ lead to a common sequence of plasma membrane injury, altered calcium homeostasis, accumulation of intracellular calcium ions, and cellular death by different active processes involving the metabolic alteration of the original chemicals. Since TRQ suppresses cellular injuries induced by both hepatotoxins, TRO may have an effect on the common mechanism, that is, alternation of calcium homeostasis.

\section{References}

1 Umezu, K., Yuasa, S. and Sudoh, A.: Antidotal properties of tritoqualine against acute liver injury by some toxic agents in rats. Folia Pharmacol. Japon. 85, 387-395 (1985) (Abs. in English)

2 Yuasa, S., Sudoh, A., Nakao, Y. and Umezu, K.: Suppressive effect of tritoqualine on lipid peroxidation and enzyme leakage induced by carbon tetrachloride in rat hepatocytes. Japan. J. Pharmacol. 41, 205-210 (1986)

3 Watanabe, A., Akamatsu, K., Takesue, A. and Takeda, K.: Dysregulation of protein synthesis in injured liver. Enzyme 23, 320-327 (1978)

4 Kiso, Y., Tohkin, M. and Hikino, H.: Assay method for antihepatotoxic activity using galactosamine-induced cytotoxicity in primarycultured hepatocytes. J. Nat. Prod. 46, 841-847 (1983)

5 Tran-Thi, T.A., Phillips, J., Falk, H. and Decker, K.: Toxicicity of D-galactosamine for rat hepatocytes in monolayer culture. Exp. Mol. Pathol. 42, 89-116 (1985)

6 Uchiyama, M. and Mihara, M.: Determination of malonaldehyde precursor in tissues by thiobarbituric acid test. Anal. Biochem. 86, 271-278 (1978)

7 Schanne, F., Pfau, R. and Farber, J.: Galactosamine-induced cell death in primary cultures of rat hepatocytes. Am. J. Pathol. 100, 25-36 (1980)

8 Yoshikawa, T., Furukawa, Y., Murakami, M.r Takemura, S. and Kondo, M.: Effects of vitamin $E$ on D-galactosamine-induced and carbon tetrachloride-induced hepatotoxicity. Digestion 25, 222-229(1982)

9 Farber, J.L.: Calcium and the mechanism of liver necrosis. Prog. Liver Dis. 7, 347-360 (1982) 